\title{
Il contenzioso nella terapia implantare: riflessioni odontoiatriche e medico-legali
}

\author{
Domenico Vasapollo ${ }^{1}$, Luca Pieraccini ${ }^{1}$, Mario Tommaso Salerno ${ }^{2}$
}

Scuola di Specializzazione in Medicina Legale, Università degli Studi di Bologna

Odontoiatra

\begin{abstract}
The Authors examine a dentistry failure due to technical error during surgical treatment and to unsuitable behaviour. The dentist didn't perform appropriate instrumental evaluations before the surgery, that would have provided useful information about bone size. The Authors analyse informed consensus in implantology and the need of clinical protocols for an acceptable and common behaviour of dental surgeons.
\end{abstract}

Keywords: medical liability, implantology, informed consent, guidelines

A legal argument in implantology therapy: dentistry and medical-legal observation. Pratica Medica \& Aspetti Legali 2007; 1(3): 127-134

\section{INTRODUZIONE}

Da oltre un decennio si sta osservando un sensibile aumento dei casi di contenzioso, giudiziario ed extragiudiziario, riguardanti l'odontoiatria, per cui l'argomento della responsabilità professionale, materia di estrema delicatezza, merita senz'altro particolare attenzione. La dimensione del problema non è sfuggita agli Ordini Provinciali dei Medici Chirurghi e degli Odontoiatri, tant'è che anche il recente codice deontologico del dicembre 2006, per la prima volta, dedica un articolo (art. 14) alla sicurezza del paziente e alla prevenzione del rischio clinico precisando l'importanza della rilevazione, segnalazione e valutazione degli errori per poter ottenere un miglioramento della qualità delle cure. Nell'articolo in questione viene sottolineato un dato estremamente importante in quanto, per raggiungere il predetto scopo, il medico deve utilizzare tutti gli strumenti disponibili per comprendere le cause dell'evento avverso e mettere in atto i comportamenti necessari per evitarne la ripetizione: «tali strumenti costituiscono esclusiva riflessione tecnico-professionale, riservata, volta alla identificazione dei rischi, alla correzione delle procedure e alla modifica dei comportamenti». In ag- giunta, all'art. 62, riguardante l'attività medicolegale, si afferma che «l'accettazione di un incarico deve essere subordinata alla sussistenza di un'adeguata competenza medico-legale e scientifica in modo da soddisfare le esigenze giuridiche attinenti al caso in esame, nel rispetto dei diritti della persona e delle norme del Codice di Deontologia Medica e preferibilmente supportata dalla relativa iscrizione allo specifico albo professionale», precisandosi in seguito che "in casi di particolare complessità clinica e in ambito di responsabilità professionale, è doveroso che il medico legale richieda l'associazione con un collega di comprovata esperienza e competenza nella disciplina coinvolta».

Lesperienza personale, frutto di una collaborazione stretta e rispettosa delle specifiche competenze di ognuno di noi, ha portato a una analisi medico-legale attenta e scrupolosa dei numerosi casi giunti alla nostra osservazione, ed è per tale motivo che oggi ne proponiamo uno, meritevole di segnalazione, in quanto riguarda la tecnica implantologica, oggigiorno utilizzata con crescente frequenza da un elevato numero di dentisti; pratica che propone, peraltro, peculiari riflessioni medico-legali essendo gravata da insuccessi se non vengono attentamente rispettate le singole fasi dei protocolli operativi. 


\section{DESCRIZIONE DEL CASO}

Per risolvere l'edentulismo del primo molare superiore destro (16), un paziente di 48 anni, previo esame radiografico, si sottopone a terapia implantare. Durante l'inserimento si verifica la migrazione di una vite nel seno mascellare di destra. Plurimi accertamenti clinico-strumentali permettono di rilevare, in particolare, lo sfondamento del pavimento mascellare, con vite disposta in cavità orizzontalmente in senso antero-posteriore, e la reazione infiammatoria della mucosa del seno. Stante il tentativo infruttuoso da parte dell'operatore di recuperare la vite, a distanza di circa un mese il paziente è sottoposto a un intervento chirurgico di rimozione, in anestesia generale, durante il quale si effettua incisione della mucosa gengivale in regione 16 , scheletrizzazione del tavolato osseo sottostante, allargamento della breccia ossea ed esplorazione del seno mascellare destro al cui interno si rinviene l'impianto dentario (poi rimosso), oltre a curettage del seno mascellare interessato da processo flogistico. In seguito è posizionato un catetere di drenaggio e viene effettuata l'asportazione del tramite fistoloso. Nel decorso successivo il paziente lamenta disturbi nasali con sensazione di ostruzione e incremento di secrezione, oltre a episodi di intenso dolore sinusale. Ai controlli clinico-strumentali si documenta, in particolare, edema della mucosa a livello della fossa nasale e velamento del seno mascellare destro, turbe della funzionalità tubarica omolaterale con modico gap trasmissionale all'audiometria. Per l'edentulismo dell'elemento 16 viene in seguito effettuata una protesi fissa ceramico-metallica su 15-16-17.

Nel corso delle indagini peritali il paziente lamenta senso di ovattamento auricolare a destra, cambiamento della percezione della voce per modificazione del timbro, oltre a disturbi sinusali caratterizzati da rinorrea e dolore puntorio intermittente in sede sottorbitale e mascellare destra con irradiazione all'emifaccia di appartenenza trattati mediante terapia farmacologica locale e sistemica. L'esame obiettivo del cavo orale rileva in particolare una cicatrice della mucosa nel vestibolo del I quadrante, oltre ad algia alla palpazione del punto di emergenza della branca mascellare del trigemino destro.

\section{GLI ASPETTI CLINICI}

L'impiego di impianti dentari come pilastri di protesi ha rappresentato per molto tempo una metodica priva di scientificità, affidata a una serie di tecniche sperimentali non sostenute da ricerche e studi clinici. L'utilizzazione di diversi materiali ha posto quesiti di tollerabilità biologica e di resistenza alla corrosione e alla rottura. Attualmen- te, in seguito ad acquisizioni relativamente recenti, la tecnica implantare appare standardizzata e viene utilizzata di frequente da un elevato numero di dentisti [1]. Gli impianti via via proposti nel corso degli anni, in relazione alla forma, possono essere schematicamente suddivisi in endodontici (non più utilizzati), inserti intramucosi (anch'essi abbandonati), sottoperiostali ed endossei. L'analisi della letteratura sull'insieme dei risultati ottenuti in passato, secondo i diversi Autori e le differenti tecniche, mostra una media di insuccessi implantari dal 40 al $70 \%$ per un periodo di osservazione compreso tra i dieci e i quindici anni. Tale dato avvalora l'ipotesi che anche un impianto parzialmente osteointegrato o fibrointegrato possa risultare clinicamente valido per molti anni. Dopo le applicazioni iniziali l'implantologia ha continuato a evolversi sotto la spinta della ricerca scientifica e industriale; gli impianti endossei hanno subito una notevole evoluzione rappresentando al momento l'applicazione più frequente e i risultati sono suffragati dagli studi clinici in modo tale da essere considerati una valida alternativa alla protesi tradizionale.

Per raggiungere un'elevata percentuale di successo è necessario rispettare le singole fasi dei protocolli operativi, standardizzati e sperimentati. Il primo è rappresentato, ovviamente, dalla selezione del paziente. A tale proposito è necessario valutare sia le condizioni di salute generale sia quelle locali delle ossa mascellari. A quest'ultimo riguardo è utile precisare che devono essere assenti infezioni delle mucose e dell'osso, ed è necessario un approfondito studio morfologico dell'osso, in quantità (altezza verticale e forma della cresta, limiti anatomici: nervo mandibolare e seni mascellari) e in qualità. Per quanto attiene le motivazioni del paziente vanno considerati candidati all'implantologia i soggetti che presentano condizioni cliniche che precludono la possibilità di applicare una protesi mobile funzionalmente valida o che rifiutino tecniche convenzionali protesiche. La successiva fase dei protocolli operativi prevede la progettazione. Gli impianti endossei, essendo tecniche di profondità, richiedono, come detto, un'adeguata quantità di osso. Le zone ideali per l'implantologia sono rappresentate dalla regione mandibolare anteriore posta tra i due forami mentonieri e la zona mascellare anteriore situata davanti ai seni mascellari, al di sotto della cavità piriforme, in pazienti edentuli con corticali ossee spesse e osso trabecolare ben calcificato. È doveroso ricordare che l'applicazione di impianti in situazioni diverse può essere gravata da maggiori percentuali di insuccesso [1]. Al contrario, secondo Misch [2], l'impianto singolo posteriore è molto più semplice da inserire e da protesizzare, rispetto al dente singolo anteriore, tant'è che afferma che «è una buona zona per iniziare a fare esperienza chirurgica e/o protesica in implantologia». Questo Autore segnala inoltre che i primi molari sono denti molto importanti per la masticazione e per il mantenimento di un appropriato schema 
occlusale e, in loro assenza, devono essere sostituiti, riportando che i vantaggi della sostituzione del dente singolo posteriore sono la maggiore larghezza ossea disponibile, il tavolato osseo vestibolare più spesso che ritarda il riassorbimento dell'osso (per cui è possibile spesso inserire impianti da 4-5 o anche $6 \mathrm{~mm}$ di diametro senza innesto), l'estetica cervicale (comprese le papille interdentali, sia a livello del contorno e della forma della corona) meno richiesta. Un ulteriore elemento a favore della tecnica implantare è la considerazione che i denti naturali adiacenti all'edentulia, quando impiegati come monconi per un ponte fisso, vanno incontro a un aumento di frattura della ceramica, ipersensibilità dentale, complicazioni endodontiche, carie e problemi parodontali. E sono proprio queste complicazioni che rappresentano la principale fonte di contenzioso (in senso assoluto) nell'ambito odontoiatrico, raggiungendo il $60 \%$ del totale dei casi. Per contro tale Autore riporta, quali svantaggi inerenti all'implantologia in regioni posteriori, le maggiori forze sviluppate durante la funzione, l'altezza limitata per l'anatomia e la variabilità della qualità ossea.

Dopo aver analizzato e considerato la zona da protesizzare, è fondamentale studiare il numero di impianti (deve esserci un rapporto di 1:1 o 1:2 con il numero di denti mancanti) ed eventuali interventi complessi da progettare in presenza di insufficiente volume osseo (rialzo del seno, arricchimenti ossei con trapianti di materiale osseo o sintetico, rigenerazione ossea con membrane, ricostruzione di cresta). A tale proposito si vuol ricordare che gli impianti singoli nelle regioni posteriori del mascellare superiore possono essere inseriti senza il rialzo del seno mascellare quando si hanno almeno $12 \mathrm{~mm}$ di osso disponibile; al diminuire della densità ossea deve corrispondere una maggiore altezza dell'osso [2]. Anche se una regola dell'odontoiatria implantare è di rimanere sempre a due millimetri di distanza dai limiti di sicurezza, questa non vale nella mascella posteriore. Tuttavia quando ci si trovi in situazioni di insufficiente qualità (minore densità) o quantità (altezza inferiore ai $12 \mathrm{~mm}$ ) di osso è possibile ricorrere, come detto, ad interventi chirurgici ausiliari (ad esempio il rialzo del seno mascellare) ${ }^{1}$. Una seconda possibilità è quella di inserire un im-

\footnotetext{
${ }^{1}$ Tale pratica operatoria viene suddivisa in piccolo rialzo e grande rialzo del seno. Nel primo l'obiettivo della chirurgia è di innalzare il seno in modo da guadagnare $2 \mathrm{~mm}$ utilizzando l'accesso dell'osteotomia implantare per raggiungere un minimo di 12 $\mathrm{mm}$ di altezza. L'impianto viene inserito contemporaneamente al procedimento di rialzo del seno. L'osteotomia viene preparata secondo gli schemi classici di preparazione e si ferma a 1- $2 \mathrm{~mm}$ al di sotto del pavimento dell'antro. Si seleziona un osteotomo piatto o arrotondato dello stesso diametro dell'osteotomia finale. L'osteotomo viene inserito e picchiettato con fermezza nella sua posizione finale, $2 \mathrm{~mm}$ oltre la preparazione effettuata. Sul pavimento antrale si crea una frattura a legno verde che solleva l'osso e la membrana sinusale grazie alla punta smussa dell'osteotomo. Il successivo inserimento dell'impianto innalza ulteriormente la membrana assieme alla copertura ossea in maniera molto graduale. La scelta del grande rialzo del seno mascellare
}

pianto endosseo ritardato, a cicatrizzazione avvenuta quando la cresta residua è inferiore a $5 \mathrm{~mm}$. In queste condizioni, l'altezza dell'osso è certamente inadeguata per poter inserire l'impianto o gli impianti con ragionevoli possibilità di successo se non contemporaneamente a un intervento di innesto osseo. Il prelievo può avvenire in ambito orale (mandibola) o extraorale (cresta iliaca, teca cranica, ecc.). La regione rialzata e innestata matura in un periodo che va da sei a dieci mesi e solo dopo questo tempo verranno inseriti impianti. Solo dopo la fase di progettazione, che come visto richiede un'attenta analisi sia delle condizioni generali del paziente che dell'anatomia scheletrica dello stesso, è possibile passare alla fase operativa-chirurgica che richiede, in particolare, la preparazione dell'alloggiamento implantare che deve risultare preciso per permettere la stabilità primaria dell'impianto, lasciando il minor spazio morto possibile per facilitare la rigenerazione ossea perimplantare. Inoltre è utile ricordare che le frese devono essere utilizzate in modo da non ledere con il calore gli osteoblasti e pertanto è preferibile impiegare quelle munite di raffreddamento interno e a 40-80 giri al minuto. Solo in seguito, a distanza di un congruo periodo di tempo, si procederà alla fase della protesizzazione $\mathrm{e}$ al successivo mantenimento. Lassenza di igiene orale potrebbe infatti essere la causa del fallimento implanto-protesico e per tale motivo il paziente deve essere edotto sulla necessità di richiami $\mathrm{e}$ controlli periodici.

Numerosi articoli in letteratura registrano per gli impianti osteointegrati percentuali di successo del 90-95\% nel mascellare superiore e del 95-98\% nella mandibola, per un periodo di osservazione variabile tra i 5 e i 10 anni. La scuola svedese riporta casistiche con una percentuale di successo inferiore, comunque superiore all' $80 \%$ sul mascellare e al $90 \%$ sulla mandibola dopo un periodo di osservazione clinica di 15 anni. I criteri di valutazione del successo implantare sono rappresentati da immobilità dell'impianto singolo alle prove cliniche, assenza di zone radiotrasparenti alle radiografie retroalveolari, assenza di segni o sintomi persistenti o irreversibili (dolore, infezione, lesioni neurologiche), perdita ossea inferiore a $0,2 \mathrm{~mm}$ all'anno dopo il primo anno.

è indicata quando l'altezza fra la cresta e il pavimento del seno è almeno di $5 \mathrm{~mm}$ e lo spessore dell'osso disponibile è maggiore di $5 \mathrm{~mm}$. La tecnica prevede una incisione crestale a tutto spessore secondo la classica Coldwell-Luc e successiva preparazione di una finestra di accesso mediante frese rotanti e abbondante irrigazione con fisiologica sterile facendo attenzione a non lesionare la membrana bluastra del seno mascellare. Con l'aiuto di uno scollaperiosteo più largo lungo il margine inferiore si stacca la finestra e si discosta la membrana nella parte inferiore del seno sempre sotto controllo visivo da parte del chirurgo. A questo punto possono essere inseriti diversi materiali di innesto che possiedono attività osteogenetica-osteoconduttiva (osso autologo, osso demineralizzato-congelato, in polvere, fosfato beta tricalcico, idrossiapatite eterologa e diverse combinazioni di queste). Inserito l'impianto, si attende un periodo di circa sei mesi per la sua integrazione [2] 
I fallimenti implantari² ${ }^{2}$ che richiedono la rimozione dell'impianto (nel 5-10\% dei casi) e che si manifestano solitamente entro il primo anno dalla protesizzazione sono dovuti tipicamente a:

- errori nella fase chirurgica che pregiudicano la realizzazione dell'osteointegrazione, ad esempio la scorretta preparazione del letto implantare o il riscaldamento eccessivo e la necrosi ossea a causa di pressione eccessiva dello strumento rotante;

- carico precoce dell'impianto;

- errato carico masticatorio per schema occlusale scorretto della protesi;

- insufficiente igiene orale.

Si possono realizzare anche infezioni dei seni mascellari quando la porzione apicale dell'impianto raggiunge la cavità nasale o il seno mascellare per una perforazione della corticale ossea ${ }^{3}$, lesioni neurologiche, essenzialmente del ramo alveolare inferiore della branca mandibolare del nervo trigemino (dovuta a un'errata ed eccessiva profondità di lavoro), perdita di supporto osseo per osteolisi infiammatoria con riassorbimento perimplantare e perdita di osso alveolare nella zona contigua.

\section{GLI ASPETTI LEGALI}

\section{DISCUSSIONE MEDICO-LEGALE DEL CASO}

Nel caso in questione l'insuccesso terapeutico (migrazione intraoperatoria di una vite implantare nel seno mascellare) è dovuto a una combinazione di fattori. Infatti, dalla visione delle pellicole radiografiche, si rileva una scarsa quantità di osso, non sufficiente a contenere una vite sicuramente più lunga del tessuto scheletrico disponibile. Probabilmente l'operatore non ha considerato il fatto che l'indagine radiologica ingrandisce l'imma-

${ }^{2}$ Gli insuccessi vengono classicamente suddivisi in precoci (durante il periodo di osteointegrazione ovvero nei primi 46 mesi) o tardivi (dopo il carico protesico). Il fallimento della riabilitazione impianto-protesica può essere dovuto a errori di mezzi (inserti inadatti per diametro, forma, lunghezza in rapporto all'anatomia del sito, utilizzo di strumentario inadeguato, ecc), di comportamento (omissione di indagini cliniche, errata valutazione nel programma di esecuzione, ecc) o errore tecnico (inadeguatezza tra impianto e protesi per numero, posizione e/o angolazione)

${ }^{3}$ Per un errore tecnico dell'operatore durante la preparazione dell'alloggio dell'impianto o per infossamento dell'impianto causato dall'osteolisi secondaria a un'infezione perimplantare. Per evitare la perforazione della corticale ossea è necessario valutare attentamente mediante radiografie, come ricordato in precedenza, il tessuto osseo disponibile, impiegando eventualmente frese standardizzate con tacche di profondità per facilitare il controllo intraoperatorio gine del 25-30\%, (sicuramente l'esecuzione di una TAC del mascellare avrebbe fornito un'immagine di dimensioni reali) e ha tenuto conto del tessuto molle, non valido ai fini della misurazione per determinare la lunghezza dell'impianto. In tale situazione era auspicabile un preliminare e necessario intervento di rialzo del seno, onde conseguire un incremento di osso per l'inserimento di un impianto di dimensioni adeguate.

Un altro fattore da considerare è che l'operatore ha preparato in eccesso con le varie frese la cavità per quanto riguarda sia la lunghezza sia lo spessore, provocando così un'iniziale comunicazione con il seno mascellare. Durante le manovre di avvitamento della fixture la vite sfuggiva inevitabilmente al suo controllo, perdendosi nella cavità del seno mascellare dato che mancava sia il frizionamento contro le pareti ossee (cosiddetto grip) che lo stop di lavoro. In tale evenienza l'utilizzo di una vite di diametro maggiore può rivelarsi utile e il più delle volte consente di risolvere il problema.

Vi è da segnalare inoltre che il tentativo, peraltro infruttuoso, come era prevedibile, da parte dell'operatore di recuperare la vite sfuggita nel seno mascellare durante l'intervento stesso ha provocato un ulteriore assottigliamento dell'osso in sede operatoria al punto tale da condizionare inevitabilmente il successivo intervento riabilitativo. Sia l'eccessivo assottigliamento della struttura ossea, sia il disagio psichico legato all'insuccesso implantare, hanno in seguito condizionato la successiva terapia di protesi tradizionale a ponte fisso con l'elemento intermedio assente (16) coinvolgente i due elementi sani adiacenti (15 e 17) alla cresta edentula.

In conclusione un'attenta analisi radiologica avrebbe permesso di rilevare l'impossibilità della protesizzazione dentale mediante impianto, almeno senza un preventivo intervento del rialzo del seno. La fase preliminare del trattamento costituisce, infatti, un momento fondamentale dell'attività sanitaria, indispensabile per il risultato positivo del successivo decorso terapeutico. Tale procedura non solo avrebbe evitato l'effettuazione di un intervento del tutto inutile e produttivo dello sfondamento del pavimento mascellare, ma avrebbe consentito la protesizzazione dell'elemento dentale senza dover ricorrere al trattamento di una protesi tradizionale a ponte fisso non desiderata dal paziente.

\section{GLI ASPETTI LEGALI CONNESSI}

Il tema della responsabilità professionale è oggigiorno più attuale che mai in considerazione dell'aumento esponenziale delle denunce nei confronti dei medici; nonostante ciò si conosce poco della reale entità e portata di questo fenomeno. Infatti i dati sulle denunce penali verso i medici non sono facilmente reperibili nei tribunali (nonostante l'attuale sistema informatico) dato che i delitti vengono registrati per tipologia di reato e non per categoria (responsabilità professionale), né l'errore me- 
dico compare tra le cause di morte dell'ICD (International Classification of Disease). In realtà sono forniti dati che fotografano situazioni molto ristrette o quelli rilasciati dalle Compagnie assicuratrici. Per certo sappiamo, comunque, che le denunce nei confronti degli odontoiatri sono notevolmente aumentate e che per le Imprese di Assicurazione si tratta di un rischio non più tarabile, non determinabile e quindi difficilmente assumibile, tant'è che alcune compagnie cercano di contenere il fenomeno attraverso la riduzione delle garanzie prestate, mentre altre fuggono dal settore della RCD (Responsabilità Civile Diversi). L'aumento delle denunce e dei premi, oltre alla riduzione delle garanzie prestate, sono, ovviamente, fatti preoccupanti per gli operatori del settore, sia da un punto di vista economico che sociale. È pur vero che un complesso di motivi, poco controllabili, contribuiscono ad alimentare e a rendere effervescente il fenomeno della responsabilità professionale. Si ricorda, ad esempio, l'interferenza dei mass-media nel loro complesso, la differente coscienza dei cittadini e la loro richiesta di salute, l'interpretazione giuridica e giurisprudenziale delle diverse fattispecie che incidono profondamente sull'andamento del fenomeno. Ad avviso di molti bisognerebbe intervenire cercando di indirizzare il cittadino verso una risoluzione extragiudiziale del contenzioso, sia attraverso una sorta di mediazione, cioè un confronto diretto con il Sanitario implicato nel presunto errore, sia attraverso una verifica della natura del reclamo, sia infine attraverso l'individuazione di un pool di esperti del settore chiamati a dare un parere tecnico di tipo medico-legale. In alcune Regioni, compresa l'Emilia Romagna, sono stati approntati alcuni progetti (protocolli integrati) per monitorare e prevenire il fenomeno della responsabilità professionale. E non è escluso che l'intero sistema, per la rilevanza che tale tematica ha assunto, sarà in futuro regolamentato da auspicabili provvedimenti legislativi come, peraltro, è già avvenuto in Svezia e in Francia. Tutto ciò al fine di ridurre i tempi di definizione del sinistro e dei conseguenti costi. Ma l'obiettivo prioritario di tutti è prevenire, o quantomeno contenere, il fenomeno della malpractice. Da alcuni anni si va affinando lo strumento di formazione e di aggiornamento dei professionisti per cercare di tradurre in termini concreti e operativi i principi del Governo Clinico (Clinical Governance), esaminando la problematica della gestione del rischio (risk management), definendo cosa significhi il termine "evento avverso", quali siano le cause, le modalità d'identificazione e le ricadute degli errori in medicina, analizzando come sia possibile imparare da essi, ossia come trasformare gli incidenti in occasioni di miglioramento della qualità del servizio. Aspetto questo sicuramente importante, dal momento che ancor oggi, specie tra i clinici, si fa molta confusione tra evento avverso, complicanza ed errore.

Lopinione rispetto ai più comuni errori in odontoiatria attualmente rimane una congettura supportata da deboli evidenze epidemiologiche, tan-

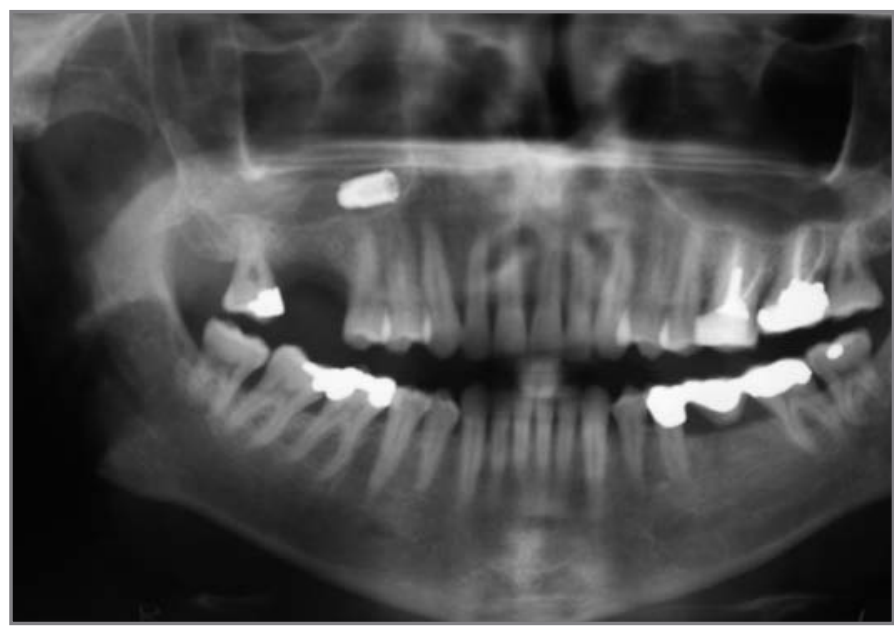

Figura 1. Vite implantare nel seno mascellare di destra

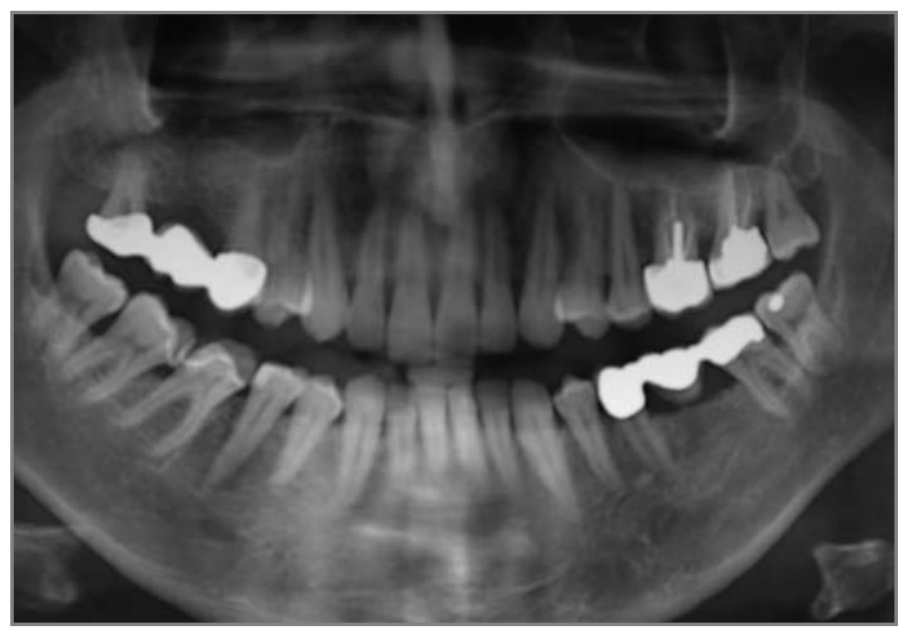

Figura 2. Protesi fissa ceramico-metallica 15-16-17

t'è che alcuni Autori hanno cercato di comprendere se la cascata degli eventi (Cascade Analisys, definita come l'analisi dell'insieme degli errori che, correlati, conducono a un incidente odontoiatrico) abbia un ruolo nel chiarire l'epidemiologia e le cause degli errori e se le relazioni mediche siano sensibili all'impatto che gli errori hanno sui pazienti. Entrando più criticamente nel problema, la ricostruzione ex post della causazione materiale, in altri termini il sapere come sono andate le cose, si deve coniugare con la dimostrazione del nesso causale materiale che si sposta sul piano della prevedibilità ex ante dell'evento e cioè della ponderazione dei rischi connessi alla prestazione per la sua specificità e/o particolare complessità e per le condizioni del paziente. Sempre a proposito della metodologia per lo studio del nesso causale, oltre alla utilizzabilità di protocolli e linee guida, va sottolineata l'importanza che si conferisce al principio della medicina basata sulle evidenze (Evidence Based Medicine). Segue, naturalmente, la disamina delle questioni attinenti alla diagnosi e alla adeguatezza del trattamento indicato ed è importante ricordare come la giurisprudenza attuale ritenga che la prestazione impianto-protesica non pos- 
sa di per sé comportare la soluzione di problemi tecnici di particolare complessità e difficoltà, dato che tale sistema è stato ampiamente studiato dalla tecnica e da tempo sperimentato nella pratica odontoiatrica, cadendo così la protezione giuridica dell'articolo 2236 c.c.

Un'altra problematica, non meno importante, è quella dell'informazione e del consenso. Gli errori di comunicazione sembrano, infatti, giocare un ruolo molto importante nella propagazione degli errori diagnostici e terapeutici per cui il miglior livello qualitativo-quantitativo d'informazione, documentando nel contempo l'avvenuta comprensione e accettazione delle procedure proposte, parrebbe un modello utile per ridurre, almeno in parte, la rivendicazione dei pazienti nei confronti dei Sanitari. Ad esempio, in alcuni Paesi, si consegna all'interessato un programma informatico attraverso il quale il paziente segue su un personal computer un percorso interattivo, strutturato anche in forma di test di verifica dell'effettivo grado di comprensione, che fornisce tutte le informazioni cliniche attinenti ai suoi problemi, opportunamente elaborati per il livello di cultura dello stesso, così da ottenere, più che un consenso informato, una scelta informata tale da rendere il paziente in grado di assumere le decisioni che meglio corrispondono ai suoi interessi. Infatti per perseguire una condotta medica adeguata, esente da censure, non basta scegliere e attuare un trattamento corretto, ma occorre evitare anche che l'eventuale danno iatrogeno, non causato da errori o da altre trasgressioni di procedure, divenga motivo di contenzioso per mancanza di un valido consenso. Non potendo trattare nei dettagli le problematiche relative a questa importante e delicata questione, su cui si sono scritti numerosi volumi, si ritiene tuttavia utile ricordare che con il termine "consenso informato" ci si riferisce alla protezione della dignità e all'autodeterminazione del paziente. Il medico ha il dovere d'informare il cittadino sia perché la mancata informazione violerebbe il dovere di comportarsi secondo buona fede durante le trattative e nella formazione del contratto, sia perché rappresenta nella sostanza l'unico modo per ottenere dal paziente un valido consenso pertinente ai principi della Costituzione (artt. 13 e 32). Il diritto all'informativa appartiene, pertanto, a quei diritti inviolabili della persona, in ordine a tutte le sfere e ambiti in cui si svolge la personalità dell'uomo, fino a comprendere anche la consapevole adesione al trattamento sanitario. Il consenso del paziente dovrà essere espresso con volontà immune da vizi, e cioè libero da coartazioni, inganni o errori. Il Sanitario, nel fornire corretta e adeguata informazione, che rappresenta l'elemento fortemente legittimante l'intervento medico, e nel rispetto del "diritto del paziente alla verità", dovrà descrivere la patologia che intende trattare, dovrà esporre caratteristiche, modalità, rischi e benefici, obiettivi del trattamento che si intende adottare, non tralasciando di menzionare le alternative eventualmente percorribili. Va, a questo punto, richiamata l'attenzione sul fatto che l'omissione della necessaria informazione è considerata dalla giurisprudenza, di per sé, fonte di responsabilità, anche in assenza di qualsivoglia errore tecnico-professionale, con addebito dell'integrale risarcimento delle conseguenze dannose subite dal paziente. I capisaldi del sistema risarcitorio del "consenso non informato", e cioè dei casi in cui un'informazione lacunosa abbia compromesso il diritto del paziente di determinarsi convenientemente rispetto alle esigenze della cura e conservazione dei principali interessi ed esigenze della vita, sono costituiti da un'informativa colpevolmente carente e concretamente incidente sulla decisione terapeutica, cui non si sarebbe consentito a fronte di un'informazione completa e corretta [7-13].

Un altro tema che si vuole sinteticamente ricordare riguarda l'interpretazione giuridica e medicolegale delle linee guida [14-29]. Infatti tale strumento, di grande utilizzo nella pratica clinica, è ritenuto da una cospicua letteratura medica assai utile per migliorare e razionalizzare l'assistenza sanitaria, riconosciuto quindi strumento perfetto in ragione dell'autorevolezza di chi le compila, della validità del metodo e altro ancora; da altri, al contrario, è considerato di scarsa rilevanza data la necessità di costante aggiornamento. Le raccomandazioni date dalle linee guida vengono comunque elaborate in modo sistematico allo scopo di fornire ai Sanitari e ai pazienti un supporto per scegliere le modalità di assistenza più appropriate in specifiche circostanze cliniche, rese opportune dalla crescente complessità del sistema sanitario e dalle tecnologie che gli sono proprie, dalla costante introduzione di nuove metodiche diagnostico-terapeutiche e da incombenti necessità economiche. Ricordando che alcuni Paesi stranieri hanno già legiferato sul tema delle linee guida, è necessario porre attenzione sul loro ruolo quando vi è una valutazione di presunta malpractice. Attualmente in Italia, da un lato sono in vigore generiche previsioni legislative sull'opportunità del ricorso alle linee guida (ad esempio il Piano Sanitario Triennale 1998/2000 del Ministero della Sanità, la Legge Finanziaria del 1998 e altre ancora), dall'altro il Codice di Deontologia Medica 2006, stabilendo che l'esercizio della medicina è fondato sulla libertà e sull'indipendenza professionale (art. 4: diritto inalienabile del medico) e che le prescrizioni e i trattamenti devono essere ispirati ad aggiornate e sperimentate acquisizioni scientifiche (art. 13), di fatto impone l'obbligo di aggiornamento e formazione professionale. Pertanto è possibile affermare che il medico deve agire con "scienza propria", ma deve anche seguire le indicazioni ufficiali, le linee guida, per distaccarsene solo quando ritenga che occorra un diverso modo di procedere. Altro aspetto fondamentale è che le linee guida sono fondate sulla malattia e non sul malato che deve essere curato e, pertanto, devono essere guardate con estrema prudenza in quanto il clinico ha il dovere di scrutare ciò che differisce dalla regola comune, dal momento che non sussistono due malati uguali. Infi- 
ne, altro punto essenziale è che non vi è uniformità di pensiero sul valore delle linee guida in tema di colpa professionale. Vi è, infatti, chi sostiene (errando) che, qualora il Sanitario non segua una linea guida e si verifichi un danno, possa configurarsi addirittura una colpa specifica e chi, al contrario, ritiene che tali strumenti non debbano essere seguiti ciecamente e che si possa configurare una colpa generica soltanto quando venga provato un comportamento non adeguato. In ogni caso, dato che l'obiettivo delle linee guida è quello di migliorare il livello e la qualità dell'assistenza, tendendo le indicazioni a un risultato clinico d'eccellenza, in ambito medico-forense esse potranno assumere il ruolo di mero riferimento, in quanto, in ogni caso, si dovrà tenere conto della diligenza "media" del professionista, stante la variabilità (bio-patologica) del soggetto e la relativa risposta terapeutica. Per tale motivo si concorda con quegli Autori che ritengono che non sia possibile individuare automaticamente una colpa professionale in caso di inosservanza delle linee guida, causativa di nocumento al paziente. Al contrario è possibile affermare che il ricorso a linee guida non adeguate possa integrare responsabilità in caso di evento dannoso, potendo il Sanitario derogare da esse. È utile, comunque, ricordare che, in assenza di consenso, il ricorso a una linea guida o la deroga dalla medesima, anche se tecnicamente giustificabili, rappresentano un trattamento sanitario abusivo, giacché il trattamento medico, incidendo su beni primari e su diritti della persona costituzionalmente garantiti, trova un limite invalicabile, come detto, nel consenso dell'avente diritto che costituisce il requisito di liceità imprescindibile del trattamento medico. In conclusione, su questo punto, in accordo con altri Autori [20] riteniamo che la linea guida andrebbe intesa come esaltazione della professionalità medica e della consapevolezza dei compiti della Medicina all'interno della società e non certamente come funzione deresponsabilizzante e di rinuncia all'individualità, alla professionalità e alla libertà della Medicina.

Un ultimo aspetto di grande attualità è la depenalizzazione della colpa professionale medica che dovrebbe essere prevista nel nuovo disegno di legge del ministro Livia Turco, dato che l'Italia è l'unico Paese, oltre al Messico, in cui è previsto il reato penale per i medici, con ricorso giudiziario estremamente frequente stante l'assenza di co- sti e la necessità per il paziente di incardinare un processo civile. In tale Decreto dovrebbe essere prevista anche la creazione di meccanismi o camere di conciliazione tra medico e paziente (invece di far scattare la denuncia penale), per la definizione stragiudiziale delle vertenze, lo spostamento sulle aziende sanitarie della responsabilità civile con assunzione del rischio al posto del professionista (in toto nella prima stesura del DDL, "in parte" nella seconda), l'istituzione di un servizio di ingegneria clinica per il controllo tecnico delle strutture e la verifica delle apparecchiature, nonché la creazione, da parte delle aziende, di forme alternative alle assicurazioni, formando realtà interne patrimoniali di fideiussione con le banche per coprire i propri rischi. Il Senatore Bodini della Commissione Sanità del Senato ha segnalato durante il Convegno di Responsabilità e Gestione del Rischio in Medicina d'Urgenza, tenutosi presso l'Ospedale George Eastman di Roma nel giugno 2007, come peraltro affermato da noi in precedenza, che «quello che manca nel DDL della Turco è l'Osservatorio del Contenzioso Medico che invece sarebbe urgente perché abbiamo un disperato bisogno di dati», oltre che l'assenza di fondi di garanzia dell'area terapeutica per risarcire i pazienti dei danni dovuti alle complicanze imprevedibili insite nelle cure stesse.

\section{CONCLUSIONI}

Gli orientamenti giurisprudenziali più attuali tendono a superare l'obsoleta tesi dicotomica della responsabilità professionale per dare maggiore risalto all'informazione e al consenso al trattamento sanitario ed evidenziano anche che la prestazione impianto-protesica non comporta la soluzione di problemi tecnici di particolare complessità e difficoltà. La diffusione delle tecniche implantologiche ha portato l'emersione di una casistica in progressiva espansione di insuccessi riguardanti prevalentemente la fase pre-chirurgica e operatoria. È interesse degli operatori di settore contribuire alla codificazione di linee guida affidabili e condivise, definendo protocolli clinici capaci di costituire un modello di comportamento accettabile.

\section{BIBLIOGRAFIA}

1. Montagna F, De Leo D, Carli PO. La responsabilità professionale odontoiatrica. Roma: Promoass, 1998

2. Misch CE. L'odontoiatria implantare contemporanea. Roma: Antonio Delfino, 1999

3. Montagna F. Le patologie professionali del personale odontoiatrico e il contenzioso odontoiatra-paziente. Rischi, prevenzione, normative e responsabilità. Milano: Masson, 2001

4. Montagna F. Testo atlante di odontoiatria medico-legale. Milano: Masson, 2005 
5. Corato A. La responsabilità professionale nella riabilitazione impianto-protesica. In: Atti del Convegno Odontoiatria e Diritto. Siena, 27-30 settembre 2000

6. Vasapollo D. La responsabilità professionale nella moderna implantologia. In: Atti del convegno nazionale della sezione di radiologia odontostomatologica e maxillo-facciale, Bologna 25-27 ottobre 2001, Tipografia Moderna Bologna, 2001

7. Cazzaniga A. Compendio di medicina legale e delle assicurazioni. Torino: Utet, 1995

8. Puccini C. Istituzioni di medicina legale. Milano: CEA, 2003

9. Santosuosso A. Il consenso informato. Milano: Cortina, 1996

10. Fucci S. Informazione e consenso nel rapporto medico-paziente. Milano: Masson, 1996

11. Passacantando G. Il consenso e i suoi riflessi sulla responsabilità penale del medico. Riv It Med Leg 1999; $21: 787$

12. Bilancetti M. Le conseguenze di rilevanza penale e civile del consenso invalido. Il consenso informato: un continente ancora da esplorare. Riv It Med Leg 2003; 6: 946-63

13. Passacantando G. Il principio del consenso e l'arbitrarietà del trattamento medico-chirurgico. Riv It Med Leg 2003; 25: 67

14. Barni M. Evidence-based Medicine e medicina legale. Riv It Med Leg 1998; 3-10

15. Fineschi V, Frati P. Linee-guida: a double-edged sword. Riflessioni medico-legali sulla esperienza statunitense. Riv It Med Leg 1998; 20: 665

16. Barni M. Le linee guida. In: Consulenza medico-legale e responsabilità medica. Milano: Giuffrè, 2002

17. Grilli R. Definizione, obiettivi, rischi e potenzialità della linea guida. In: Atti del convegno "Regole e libertà di cura: implicazioni sociali, professionali e medico-legali nelle linee guida cliniche”. Perugia, 27-28 marzo 1998

18. Musicco M. Le linee guida tra evidenza scientifica e buon senso. Medic 2000; 8: 67

19. Lafisca $S$, Selle V, Mariotto A. Le linee guida nella pratica medica: considerazioni medico legali e di sanità pubblica. Professione 1996; 5: 54

20. Norelli GA, Mazzeo E, Pinchi V. Le linee guida offrono al professionista una copertura medico-legale? Professione Sanità pubblica e medicina pratica 1988: $11 ; 31$

21. Woolf SH. Practice guidelines: a new reality in medicine. Arch Intern Med 1993; 153: 2646

22. Wolff SH. Practice parameters in health reform: new state approaches precede Clinton plan. J Law Med Ethics 1993; 21: 394

23. Merrit TA, Palmer D, Bergman DA, Shiono PH. Clinical practice guidelines in paediatric and newborn medicine: implication for their use in practice. Pediatrics 1997; 99: 100

24. Kane RL. Creating practice guidelines: the dangers of over-reliance on expert judgment. J Law Med Ethics 1995; 23: 62

25. Federspil G, Vettor R. I limiti della medicina: probabilità, errori e linee-guida. In: Il rischio in Medicina oggi e la Responsabilità professionale. Atti del Convegno di Studio Roma, 26 giugno 1999. Milano: Giuffrè, 2000

26. Barni M. Responsabilità del medico tra deontologia e diritto. In: Il rischio in Medicina oggi e la Responsabilità professionale. Atti del Convegno di Studio Roma, 26 giugno 1999. Milano: Giuffrè, 2000

27. Giannini R. Linee guida in sanità: gli aspetti medico-legali. Salute e territorio 1997; 104: 255

28. Turillazzi E. Evidence Based Medicine e professione. Professione. Cultura e pratica del medico d'oggi 2002; 9: 30-7

29. Cicognani A. Linee Guida. In: Atti del Convegno "Problematiche medico legali in cardiologia invasiva”, Bologna, 22 novembre 2002

30. Vasapollo D, Pieraccini L. Osservanza delle linee guida e prospettive medico-legali - prima parte. ANDI notizie 2003; $149: 7$

31. Vasapollo D, Pieraccini L. Osservanza delle linee guida e prospettive medico-legali - seconda parte. ANDI notizie 2004; 150: 5

32. Aspriello SD, Badolato A, Piemontese M. Impianto-protesi: dall'insuccesso terapeutico al concorso di responsabilità professionale. Jura Medica 2006; 3: 585

\section{CORRESPONDING AUTHOR}

Prof. Domenico Vasapollo, e-mail: domenico.vasapollo@unibo.it 